\title{
HIGHLIGHTS
}

GENETICS

\section{Staging and prognostic value of p16 expression in oropharyngeal cancer}

A strong independent prognostic marker for oropharyngeal squamous cell carcinoma (OPSCC) has been identified by researchers in Switzerland. The report suggests that expression of p16 should be included in the official staging system for head and neck squamous cell carcinoma (HNSCC) to identify patients with OPSCC. Claude Fischer and colleagues found that p16 was a stronger and more reliable prognostic factor than conventional staging parameters.

Currently, primary tumor extension, lymph-node involvement and distant metastasis are used to determine the stage of HNSCC. A step towards improved differentiation of HNSCC into distinct subgroups was achieved by the identification of human papillomavirus 16 and 18 in a subset of OPSCCs, which has a distinctive p16 expression profile.

Fischer et al. retrospectively evaluated the prognostic value of p16 expression relative to the established prognostic markers in 102 patients with OPSCC. The group found that $\mathrm{p} 16$ expression was a strong prognostic factor in patients with
OPSCC. "Multivariate analysis reveal[ed] p16 to be the strongest and most reliable prognostic factor compared [with] the conventional staging parameters [primary tumor extension] and [lymph node involvement] classification," Fischer reports.

The investigators note that immunohistochemical staining of p16 is relatively easy and cheap to perform compared with in situ hybridization of human papillomavirus. In addition, interpretation of p16 stained tissue samples is facilitated by the fact that any positive staining ( $>5 \%$ of tumor cells) is classified as a 16 positive tumor.

"As a first step, p16 expression should be reported regularly in prospectivelyfollowed HNSCC patients in the daily clinical setting," comments Fischer. He also recommends that the impact of the conventional prognostic staging parameters can be compared separately among the p16-positive and p16-negative OPSCCs. Finally, he proposes that p16 expression status could be introduced in a stepwise manner in clinical decision

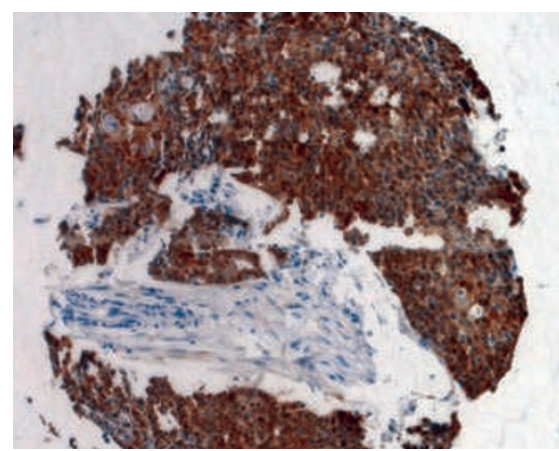

p16 stained OPSCC sample with positive staining (blue) of $100 \%$ of tumor cells. Image provided by C. A. Fischer.

making. "For example, stage III OPSCC, where an adjuvant radiotherapy after radical surgery could be indicated, should be followed very closely in a 'watch and wait' program instead of a possible adjuvant overtreatment, which is surely appropriate for conventional p16-negative HNSCC."

\section{Lisa Richards}

Original article Fischer, C.A. et al. p16 expression in oropharyngeal cancer: its impact on staging and prognosis compared with the conventional clinical staging parameters. Ann. Oncol. doi:10.1093/annonc/mdq210 